\title{
ON THE EXTENSION OF THE GAUSS MEAN-VALUE THEOREM TO CIRCLES IN THE NEIGHBORHOOD OF ISOLATED SINGULAR POINTS OF HARMONIC FUNCTIONS
}

\author{
BY G. E. RAYNOR
}

1. Introduction. Let $f(x, y)$ be a function harmonic in a plane region $R$ except at an isolated singular point $P$ in $R$, and let $C_{1}$ be a circle in $R$ with radius $r_{1}$ and with $P$ as center. In previous papers* the writer has shown that in this neighborhood $f(x, y)$ can be put in the form

$$
f(x, y)=c \log \frac{1}{r}+\Phi(x, y)+V(x, y),
$$

where

$$
c=\frac{1}{2 \pi} \int_{C_{1}} \frac{\partial f}{\partial n} d s,
$$

$r$ being the distance from $(x, y)$ to $P, \Phi(x, y)$, unless it be identically zero, harmonic in the neighborhood of $P$ and such that there exist modes of approach to $P$ for which the sum $c \log (1 / r)+\Phi$ tends toward plus infinity and also toward minus infinity; and $V$ is harmonic everywhere in the neighborhood of $P$ including $P$. Also on $C_{1}, \Phi \equiv 0$. It is to be noticed that the constant $c$ may be zero so that $\Phi$ has the same properties ascribed to the sum $c \log (1 / r)+\Phi$.

If a system of polar coordinates $(r, \theta)$ be chosen with $P$ as pole, $\Phi$ may be expanded, for $r \leqq r_{1}$, in the form $\ddagger$

* G. E. Raynor, Isolated singular points of harmonic functions, this Bulletin, vol. 32 (1926), p. 543, and Integro-differential equations of the Bôcher type, this Bulletin, vol. 32, p. 654 .

$\dagger$ Here, as in all that follows, the normal derivatives are to be taken in the direction of the inner normal.

$\ddagger$ G. E. Raynor, Note on the expansion of harmonic functions in the neighborhood of isolated singular points, Annals of Mathematics, vol. 31 (1930), p. 40. We shall refer to this as paper (A). 
(2) $\Phi=\sum_{m=1}^{\infty} \frac{1}{m}\left[\left(\frac{r_{1}}{r}\right)^{m}-\left(\frac{r}{r_{1}}\right)^{m}\right]\left(\gamma_{m} \cos m \theta+\delta_{m} \sin m \theta\right)$

where

(3) $\gamma_{m}=\frac{r_{1}}{2 \pi} \int_{-\pi}^{\pi} \frac{\partial \Phi_{r_{1}}}{\partial n} \cos m \theta d \theta ; \quad \delta_{m}=\frac{r_{1}}{2 \pi} \int_{-\pi}^{\pi} \frac{\partial \Phi_{r_{1}}}{\partial n} \sin m \theta d \theta$.

Also the two series

$$
G\left(\frac{r}{r_{1}}, \theta\right)=-\sum_{m=1}^{\infty} \frac{1}{m}\left(\frac{r}{r_{1}}\right)^{m}\left(\gamma_{m} \cos m \theta+\delta_{m} \sin m \theta\right)
$$

and

$$
G\left(\frac{r_{1}}{r}, \theta\right)=\sum_{m=1}^{\infty} \frac{1}{m}\left(\frac{r_{1}}{r}\right)^{m}\left(\gamma_{m} \cos m \theta+\delta_{m} \sin m \theta\right)
$$

are convergent for all values of $\theta$ and of $r \leqq r_{1}, *$ and $\Phi$ can be expressed in the form

$$
\Phi=G\left(\frac{r}{r_{1}}, \theta\right)+G\left(\frac{r_{1}}{r}, \theta\right) .
$$

Furthermore

$$
G\left(\frac{r}{r_{1}}, \theta\right)=\frac{r_{1}}{4 \pi} \int_{-\pi}^{\pi} \frac{\partial \Phi_{r_{1}}}{\partial n} \log \left[1-2 \frac{r}{r_{1}} \cos (\alpha-\theta)+\frac{r^{2}}{r_{1}^{2}}\right] d \alpha,
$$

which gives a solution of the Neumann problem for the circle $C_{1}$ with boundary values of the normal derivative equal to onehalf the value of the normal derivative of $\Phi$ on $C_{1} . \dagger$ The function $\Phi$ also possesses the property

$$
\int_{C} \Phi d s=0
$$

where $C$ is any circle concentric with $C_{1}$ and of radius $r \leqq r_{1}$. In view of this last property it becomes of interest to inquire as to the value of

* Paper (A), p. 40. Note that the definition of $G\left\{r / r_{1}\right\}$ of (21) of paper (A) has been slightly changed by inserting a minus sign in the right side of (4).

† Paper (A), p. 41; and Goursat, Cours d'Analyse Mathématique, vol. 3, 3d ed., p. 240. 


$$
\int_{C_{2}} \Phi d s
$$

if $C_{2}$ lies within $C_{1}$ but does not have its center at $P$. Of course, if $Q$ be the center of $C_{2}$ and $P$ lies without $C_{2}$ we have by the Gauss mean-value theorem*

$$
\frac{1}{2 \pi r_{2}} \int_{C_{2}} \Phi d s=\Phi(Q),
$$

where $r_{2}$ is the radius of $C_{2}$. Our purpose then, in this note, is to find the value of

$$
\frac{1}{2 \pi r_{2}} \int_{C_{2}} \Phi d s,
$$

in the case of $P$ within $C_{2}$. In $\$ 3$ we shall also examine the mean value of $f(x, y)$ over $C_{2}$.

2. The Mean Value of $\Phi$. Let $a$ be the distance of $Q$ from $P$ and choose the line $P Q$ as polar axis. Then by (2)

$$
\Phi(a, \theta)=\sum_{m=1}^{\infty} \frac{1}{m}\left[\left(\frac{r_{1}}{a}\right)^{m}-\left(\frac{a}{r_{1}}\right)^{m}\right] \gamma_{m}
$$

and hence by (4)

$$
G\left(\frac{a}{r_{1}}, \theta\right)=-\sum_{m=1}^{\infty} \frac{1}{m}\left(\frac{a}{r_{1}}\right)^{m} \gamma_{m} .
$$

By Green's formula, we have for the region bounded by the circles $C_{1}$ and $C_{2}$,

$$
\begin{gathered}
\int_{C_{1}}\left(\Phi \frac{\partial \log r}{\partial n}-\log r \frac{\partial \Phi}{\partial n}\right) d s \\
+\int_{C_{2}}\left(\Phi \frac{\partial \log r}{\partial n}-\log r \frac{\partial \Phi}{\partial n}\right) d s=0,
\end{gathered}
$$

where $r$ is the distance from a variable point $(x, y)$ on $C_{1}$ or $C_{2}$ to the center $Q$ of $C$, and the normal derivatives are taken

* For a statement of the Gauss mean value theorem see Goursat, loc. cit., p. 181 . 
toward the interior of our region. Now on $C_{1}, \Phi \equiv 0$ and on $C_{2}$, $\partial \log r / \partial n=1 / r_{2}$. Furthermore

$$
\int_{C_{2}} \frac{\partial \Phi}{\partial n} d s=0 . *
$$

Hence, since $\log r$ is constant on $C_{2}$, relation (10) above reduces to

$$
\frac{1}{r_{2}} \int_{C_{2}} \Phi d s=\int_{C_{1}} \log r \frac{\partial \Phi}{\partial n} d s,
$$

or

$$
\frac{1}{2 \pi r_{2}} \int_{C_{2}} \Phi d s=\frac{r_{1}}{2 \pi} \int_{-\pi}^{\pi} \log r \frac{\partial \Phi_{r_{1}}}{\partial n} d \theta .
$$

Now from (1) we have

$$
\frac{\partial f_{r_{1}}}{\partial n}=\frac{c}{r_{1}}+\frac{\partial \Phi_{r_{1}}}{\partial n}+\frac{\partial V_{r_{1}}}{\partial n} .
$$

Since $f$ is harmonic on $C_{1}$ the left side of (13) has a derivative with respect to $\theta$. Now $V$ may be written as a Poisson integral and this integral may be expressed as the sum of a constant and the potential of a double layer. Since the values of $V$ on $C_{1}$ are the values of $f$ on $C_{1}$ diminished by the constant $c \log \left(1 / r_{1}\right)$ the density of this double layer is analytic on $C_{1}$ and hence $V$ is analytic in the closed region bounded by $C_{1}$ and therefore the third term on the right of (13) has a derivative with respect to $\theta . \dagger$ Since the same is true, obviously, of the first term it follows that the second term $\partial \Phi_{r_{1}} / \partial n$ is also differentiable with respect to $\theta$ and hence is of bounded variation. $\partial \Phi_{r_{1}} / \partial n$ thus satisfies the conditions for expansion in a Fourier series and furthermore this series will be uniformly convergent in the closed interval $-\pi$ to $\pi$. We thus have

$$
\frac{\partial \Phi_{r_{1}}}{\partial n}=\frac{2}{r_{1}} \sum_{m=1}^{\infty}\left(\gamma_{m} \cos m \theta+\delta_{m} \sin m \theta\right),
$$

where $\gamma_{m}$ and $\delta_{m}$ are as given in (3). The constant term in the expansion of $\partial \Phi_{r_{1}} / \partial n$ is zero since as stated previously

* See the second paper of the first footnote.

$\dagger$ Encyklopädie der Mathematischen Wissenschaften, vol. 2, 3, 1, p. 206. 


$$
\int_{C_{1}} \frac{\partial \Phi_{r_{1}}}{\partial n} d s=0
$$

It may be pointed out that (14) may be obtained by differentiating (2) in the direction of the inner normal and then allowing $r$ to approach $r_{1}$.

Since $\log r$ is bounded on $C_{1}$ it follows from (14) that the series

$$
\log r \frac{\partial \Phi_{r_{1}}}{\partial n}=\frac{2}{r_{1}} \sum_{m=1}^{\infty}\left(\gamma_{m} \log r \cos m \theta+\delta_{n} \log r \sin m \theta\right)
$$

is uniformly convergent on $C_{1}$. Hence the series may be integrated termwise and we have

$$
\begin{array}{r}
\frac{r_{1}}{2 \pi} \int_{-\pi}^{\pi} \log r \frac{\partial \Phi_{r_{1}}}{\partial n} d \theta=\frac{1}{\pi} \sum_{m=1}^{\infty}\left[\gamma_{m} \int_{-\pi}^{\pi} \log r \cos m \theta d \theta\right. \\
\left.+\delta_{m} \int_{-\pi}^{\pi} \log r \sin m \theta d \theta\right]
\end{array}
$$

But

$$
r^{2}=r_{1}^{2}-2 r_{1} a \cos \theta+a^{2}
$$

and hence

$$
\log r=\log r_{1}+\frac{1}{2} \log \left(1-2 \frac{a}{r_{1}} \cos \theta+\frac{a^{2}}{r_{1}}\right) .
$$

Now*

$$
\int_{-\pi}^{\pi} \log \left(1-2 \frac{a}{r_{1}} \cos \theta+\frac{a^{2}}{r_{1}^{2}}\right) \cos m \theta d \theta=-\frac{2 \pi}{m}\left(\frac{a}{r_{1}}\right)^{m} .
$$

Also

$$
\int_{-\pi}^{\pi} \log \left(1-2 \frac{a}{r_{1}} \cos \theta+\frac{a^{2}}{r_{1}{ }^{2}}\right) \sin m \theta d \theta=0 .
$$

Hence by (17), (18), and (19), equation (16), since $\log r_{1}$ is a constant, takes the form

* Edwards, The Integral Calculus, vol. 2, p. 306, formula (10). The integrand in equation (18) above takes the same value in the interval $-\pi$ to 0 as in the interval 0 to $\pi$ and hence Edwards' result must be multiplied by 2 . 


$$
\frac{r_{1}}{2 \pi} \int_{-\pi}^{\pi} \log r \frac{\partial \Phi_{r_{1}}}{\partial n} d \theta=-\sum_{m=1}^{\infty} \frac{1}{m}\left(\frac{a}{r_{1}}\right)^{m} \gamma_{m}=G\left(\frac{a}{r_{1}}, \theta\right)
$$

Thus equation (12) combined with (20) gives

$$
\frac{1}{2 \pi r_{2}} \int_{C_{2}} \Phi d s=G\left(\frac{a}{r_{1}}, \theta\right)
$$

and we can state the following theorem.

THEOREM 1. The mean value of the function $\Phi$ of equation (1) over a circle $C_{2}$ within $C_{1}$ having the singular point $P$ in its interior is equal to the value of the function $G\left\{\left(r / r_{1}\right), \theta\right\}$ at $Q$, where $Q$ is the center of $C_{2}$.

Since $G\left\{\left(r / r_{1}\right), \theta\right\}$ is harmonic everywhere within $C_{2}$ its value at $Q$ by Gauss's theorem is its mean value over $C_{2}$ and hence Theorem 1 can be stated in the form:

THEOREM 2. The mean value of the function $\Phi$ over a circle $C_{2}$ within $C_{1}$, having the singular point $P$ in its interior is equal to the mean value of the function $G\left\{\left(r / r_{1}\right), \theta\right\}$ over $C_{2}$.

Since

$$
\Phi(r, \theta)=G\left(\frac{r}{r_{1}}, \theta\right)+G\left(\frac{r_{1}}{r}, \theta\right),
$$

it follows from Theorem 2 that we have the result:

TheOREM 3. The mean value of the function $G\left\{\left(r_{1} / r\right), \theta\right\}$ over a circle $C_{2}$ within $C_{1}$, having $P$ in its interior, is zero.

It is to be noticed that the above theorems are true if the center $Q$ of $C$ coincides with $P$.

3. The Mean Value of $f(x, y)$. If we wish to find the mean value of $f(x, y)$ over $C_{2}$ we must add to the mean value of $\Phi$ the mean values over $C_{2}$ of the first and third terms of equation (1). Now the first term $c \log (1 / r)$ is readily seen from (17) to be equivalent to

$$
-c \log r_{1}-\frac{c}{2} \log \left(1-2 \frac{a}{r_{1}} \cos \theta+\frac{a^{2}}{r_{1}^{2}}\right) .
$$


But we have*

$$
\int_{-\pi}^{\pi} \log \left(1-2 \frac{a}{r_{1}} \cos \theta+\frac{a^{2}}{r_{1}^{2}}\right) d \theta=0 .
$$

Thus the mean value of the first term of (1) over $C_{2}$ is $-c \log r_{1}$. Now the third term $V(x, y)$ is harmonic in $C_{2}$ and as stated previously is equal to

$$
U(x, y)+c_{1} \log r_{1},
$$

where $U(x, y)$ takes the same values on $C_{1}$ as $f(x, y)$. Hence the mean value of $V(x, y)$ over $C_{2}$ is equal to

$$
U(Q)+c_{1} \log r_{1}
$$

We thus find the mean value of the sum of the first and third terms of (1) to be $U(Q)$. Combining this result with the theorem of the previous section we have the theorem:

THEOREM 4. The mean value of the function $f$ of equation (1) over $C_{2}$ is equal to $U(Q)$ plus the value of the function

$$
G\left(\frac{r}{r_{1}}, \theta\right)=\frac{r_{1}}{4 \pi} \int_{-\pi}^{\pi} \frac{\partial \Phi_{r_{1}}}{\partial n} \log \left[1-2 \frac{r}{r_{1}} \cos (\alpha-\theta)+\frac{r^{2}}{r_{1}^{2}}\right] d \alpha
$$

at $Q$, where $U$ is the function which solves the Dirichlet problem $\dagger$ for $C_{1}$ with boundary values $f$, and $G\left\{\left(r / r_{1}\right), \theta\right\}$ is a solution of the Neumann problem for $C_{1}$ with boundary values of the normal derivatives equal to one-half the normal derivatives of $\Phi$ on $C_{1}$.

It is to be noticed that if $P$ is not a singular point, the above theorem reduces to the Gauss mean-value theorem.

The University of OKLahoma

* Edwards, loc. cit., p. 306, formula (9). The integrand in (22) above takes the same values in the interval $-\pi$ to 0 as in the interval 0 to $\pi$ and hence Edwards' result gives zero for (22).

$\dagger$ For a statement of the Dirichlet problem see Goursat, loc. cit., p. 196. 